\title{
Lubrication of Isotropic Permeable Porous Inclined Slider Bearing with Slip Velocity and Squeeze Velocity
}

\author{
Rajesh C. Shah ${ }^{1, *}$, M. M. Parsania ${ }^{2}$, H. R. Kataria ${ }^{3}$ \\ ${ }^{1}$ Department of Applied Mathematics, Faculty of Technology and Engineering, The M. S. University of Baroda, Vadodara, 390 001, \\ Gujarat State, India \\ ${ }^{2}$ Department of Mathematics, School of Engineering, R.K. University, Rajkot, Gujarat State, India \\ ${ }^{3}$ Department of Mathematics, Faculty of Science, The M. S. University of Baroda, Vadodara, 390 005, Gujarat State, India \\ nayan.patel17883@gmail.com,hrkmaths@yahoo.com
}

\begin{abstract}
This paper theoretically discusses about the inclined slider bearing with porous layer ( plates or matrix ) attached to slider as well as stator including effects of slip velocity, and squeeze velocity. General Reynolds's equation which is useful for calculating load capacity is obtained. The load capacity is calculated for isotropic case for various porosities of the above and below porous matrix. Various sizes of the porous matrix at both the ends are also discussed for the possible optimization of bearing performance. From the results we conclude that better load capacity is obtained when the thicknesses of both the porous plates are small and porosity is less. It can also be concluded from results that for smaller thickness of the porous layer and lesser porosity the load capacity increased $1.5 \%$.
\end{abstract}

Keywords Porosity, Inclined Slider Bearing, Isotropic Permeability, Slip Velocity

\section{Introduction}

In an innovative analysis $\mathrm{Wu}[1]$, dealt with the case of squeeze film behavior for porous annular disks in which he showed that owing to the fact that fluid can flow through the porous material as well as through the space between the bounding surfaces, the performance of a porous walled squeeze film can differ substantially from that of a solid walled squeeze film. The above analys is of $\mathrm{Wu}[1]$ extended by Sparrow et. al.[2] by introducing the effect of velocity slip to porous walled squeeze film with porous matrix appeared in the above plate. They found that the load capacity decreases due to the effect of porosity and slip. Prakash and Vij[3] investigated a porous inclined slider bearing and found that porosity caused decrease in the load capacity and friction, while it increased the coefficient of friction. Recently, Shah et. al.[4] studied mathematical modelling of slider bearing of various shapes with combined effects of porosity at both the ends, anisotropic permeability, slip velocity, and squeeze velocity. Many other authors have also worked in this direction, for example Kulkarni et. al.[5], Patelet.al.[6], Gupta et. al.[7], Naduvinamaniet. al.[8], Guo et. al.[9], H. Urreta et. al.[10].

In all above investigations, none of the authors in their

* Corresponding author:

dr_rcshah@yahoo.com (Rajesh C. Shah)

Published online at http://journal.sapub.org/ajfd

Copyright (C) 2012 Scientific \& Academic Publishing. All Rights Reserved study considered effects of isotropic permeability with the different porosities in both porous plates bearing. The porous layer in the bearing is considered because of its advantageous property of self lubrication. With this motivation the aim of the present work is to study the behavior of a inclined slider bearing with porous slider as well as stator including slip velocity, and squeeze velocity with effects of isotropic permeability with different porosities. The squeeze velocity is because when the upper plate approaches to lower one.

A lubrication Reynolds's equation is derived for the above system and the load capacity is calculated for isotropic permeability and different porosities at both the ends.

\section{Derivation of the Mathematical Model}

Figure 1 shows schematic diagram of a bearing with porous slider (Length $A$ in the $x$-direction and width $B$ in $y$-direction, $A<<B$ ) and inclined porous stator under study which consists of a fluid (lubricating oil) film region of thickness $h$. The value of $h$ is $h_{2}$ at the inlet and $h_{1}$ at the outlet. This film thickness $h$ is given by

$$
h=h_{2}-\left(h_{2}-h_{1}\right) x / A ; 0 \leq x \leq A
$$

Porous matrix of thickness $l_{2}$ and $l_{1}$ metres have attached with the slider and stator respectively. Both the porous matrix are backed by a solid wall. The slider moves with a uniform velocity $U$ in the $x$-direction. Also, stator moves normally towards the slider with a uniform velocity $\dot{h}=d h / d t$, where $t$ is time in seconds. 


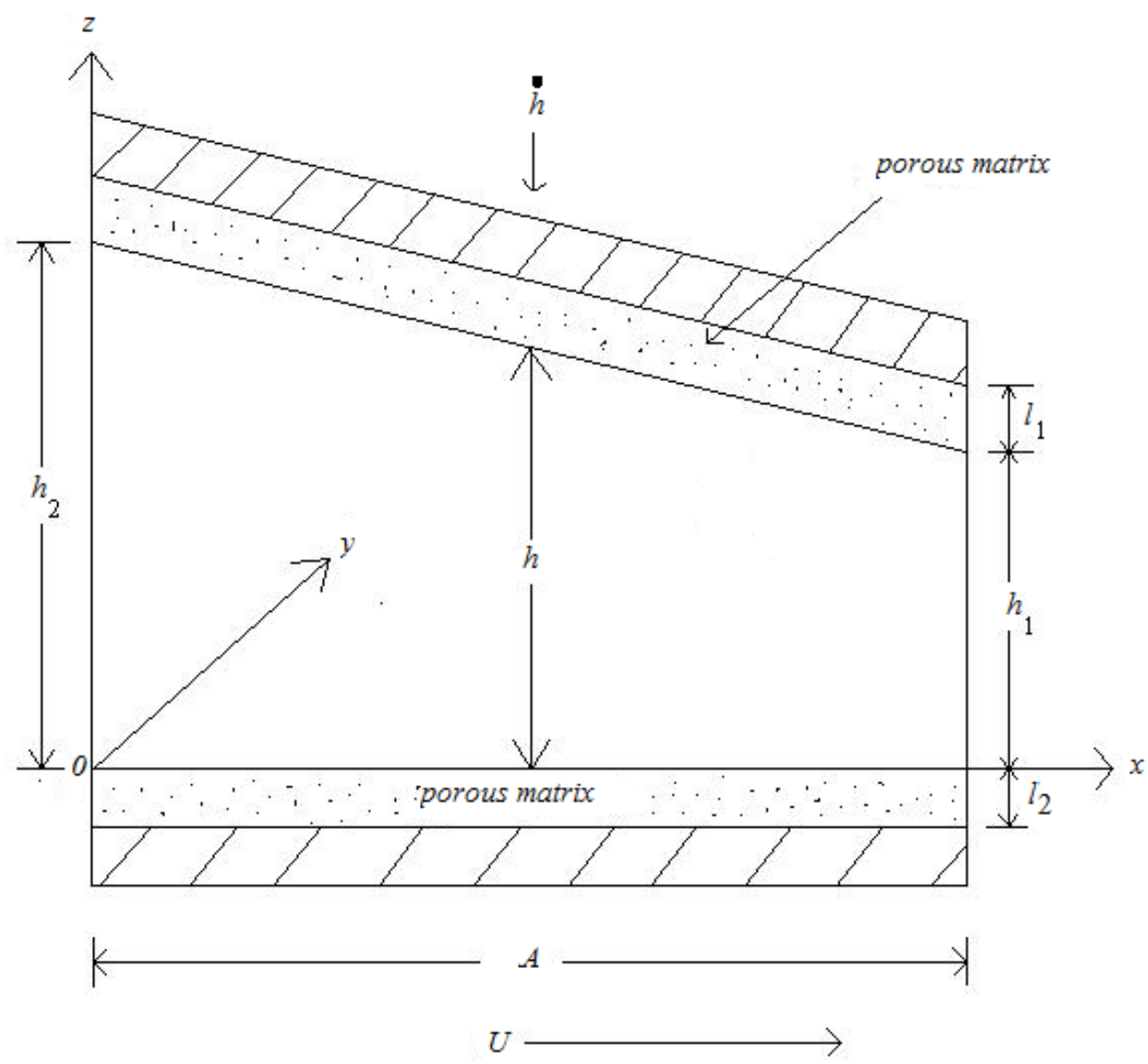

Figure 1. Inclined pad slider bearing

The basic flow equations of the above phenomenon are as follows:

(1) The one dimensional flow equation governing the lubricant flow in the film region for the above phenomenon follows form Navier-Stokes's equation under the usual assumption of lubrication, neglecting inertia terms and that the derivatives of velocities across the film predominate, yields

$$
\frac{\partial^{2} u}{\partial z^{2}}=\frac{1}{\eta} \frac{\partial p}{\partial x},
$$

where $u$ is the film fluid velocity in the $x$-direction, $p$ is film pressure there and $\eta$ is fluid viscosity.

(2)The integral form of continuity equation in the film region is given by

$$
\frac{\partial}{\partial x} \int_{0}^{h} u d z+w_{h}-w_{0}=0
$$

where $w$ is the axial component of the fluid velocity in the film.

(3) Using Darcy's law, the velocity components of the fluid in the porous matrix are given as follow:

For upper porous region:

$$
\begin{aligned}
& \bar{u}_{1}=-\frac{k}{\eta} \frac{\partial P}{\partial x}(x-\text { direction }) \\
& \bar{w}_{1}=-\frac{k}{\eta} \frac{\partial P}{\partial z}(z-\text { direction })
\end{aligned}
$$

For lower porous region:

$$
\begin{aligned}
& \bar{u}_{2}=-\frac{k}{\eta} \frac{\partial P}{\partial x}(x-\text { direction }) \\
& \bar{w}_{2}=-\frac{k}{\eta} \frac{\partial P}{\partial z}(z-\text { direction })
\end{aligned}
$$

where $k$ is fluid permeability in both the porous region in $x$ and $z$ directions respectively, and $P$ is the fluid pressure in the porous region.

(4) The continuity equation in the porous region are given as follows:

For upper porous region:

$$
\frac{\partial \bar{u}_{1}}{\partial x}+\frac{\partial \bar{w}_{1}}{\partial z}=0
$$

For lower porous region:

$$
\frac{\partial \bar{u}_{2}}{\partial x}+\frac{\partial \bar{w}_{2}}{\partial z}=0 \text {. }
$$

Solving equation (2) under the slip boundary conditions given by Sparrow et.al.[2] and modified by Shah et.al.[11] with the addition of slider velocity $U$ to[2]

$$
\begin{aligned}
& u=\frac{1}{s_{1}} \frac{\partial u}{\partial z}+U \text { when } z=0, \\
& u=-\frac{1}{s_{2}} \frac{\partial u}{\partial z} \text { when } z=h,
\end{aligned}
$$

where 


$$
\frac{1}{s_{1}}=\frac{\sqrt{k \eta_{x}}}{5}, \frac{1}{s_{2}}=\frac{\sqrt{k m_{x}}}{5},
$$

$1 / s_{i}(i=1,2)$ being slip parameter, $\eta_{x}, m_{x}$ are porosities in the $x$-direction for the lower and upper porous regions, we obtain

$$
\begin{aligned}
u=\frac{1}{\eta}\left[\frac{z^{2}}{2}-\frac{s_{1} s_{2} h z}{s}\left(\frac{h}{2}+\frac{1}{s_{2}}\right)\right. & \left.-\frac{h s_{2}}{s}\left(\frac{h}{2}+\frac{1}{s_{2}}\right)\right] \frac{\partial p}{\partial x} \\
& +\frac{s_{1} s_{2}}{s}\left[\left(\frac{1}{s_{2}}+h\right)-z\right] U^{\prime}
\end{aligned}
$$

where

$$
s=s_{1}+s_{2}+h s_{1} s_{2} .
$$

Substituting the above value of $u$ in the integral form of continuity equation (3), we have

$$
\frac{\partial}{\partial x}\left[\begin{array}{rl}
\frac{1}{\eta}\left\{\frac{h^{3}}{6}-\frac{s_{1} s_{2} h^{3}}{2 s}\left(\frac{h}{2}+\frac{1}{s_{2}}\right)\right. & \left.-\frac{s_{2} h^{2}}{s}\left(\frac{h}{2}+\frac{1}{s_{2}}\right)\right) \frac{\partial p}{\partial x} \\
& \left.+\frac{s_{1} s_{2} h U}{s}\left(\frac{1}{s_{2}}+\frac{h}{2}\right)\right]+w_{h}-w_{0}=0 .
\end{array}\right.
$$

Substituting equations (4) and (5) in the continuity equation for upper porous region (8) and then integrating the result with respect to $z$ across the upper porous matrix $\left(h, h+l_{1}\right)$, we obtain

$$
\left.\frac{\partial P}{\partial z}\right|_{z=h}=\frac{\partial^{2} p}{\partial x^{2}} l_{1},
$$

using Morgan-Cameron approximation[8] and the fact that the surface $z=h+l_{1}$ is non-porous.

Again, substituting equations (6) and (7) in the continuity equation for lower porous region (9) and then integrating the result with respect to $z$ across the lower porous matrix $\left(-l_{2}, 0\right)$, we obtain

$$
\left.\frac{\partial P}{\partial z}\right|_{z=0}=-\frac{\partial^{2} p}{\partial x^{2}} l_{2},
$$

using Morgan-Cameron approximation[8] and the fact that the surface $z=-l_{2}$ is non-porous.

Considering the continuity of the normal component of

flu id velocity across the film porous interface, so that

$$
w_{h}=\dot{h}+\bar{w}_{1}, \quad w_{0}=\bar{w}_{2}
$$

using equations (12) - (14), we obtain

$$
\begin{gathered}
\frac{\partial}{\partial x}\left(g \frac{\partial p}{\partial x}\right)=\frac{\partial f}{\partial x}, \\
\text { where } \\
g=\frac{1}{12 \eta s}\left[h^{2}\left(12+4 h s_{1}+4 h s_{2}+h^{2} s_{1} s_{2}\right)+12 s\left(k l_{1}+k l_{2}\right)\right], \\
f=\frac{s_{1} U h}{2 s}\left(2+h s_{2}\right)+x \dot{h},
\end{gathered}
$$

which is the Reynolds's type lubrication equation for the considered phenomenon.

Introducing the dimensionless quantities

$$
\begin{aligned}
& X=\frac{x}{A}, \bar{h}=\frac{h}{h_{1}}, \bar{k}=\frac{k}{h_{1}^{2}}, \overline{l_{i}}=\frac{l_{i}}{h_{1}}, \overline{s_{1}}=s_{1} h_{1}, \\
& \bar{s}_{2}=s_{2} h_{1}, \bar{p}=\frac{h_{1}^{2} p}{\eta A U}, S=\frac{-2 \dot{h} A}{U h_{1}} ; i=1,2 ;
\end{aligned}
$$

equation (1) becomes

$$
\bar{h}=a-(a-1) X ; 0 \leq X \leq 1,
$$

where

$$
a=\frac{h_{2}}{h_{1}} .
$$

Also, equation (15) transforms to

$$
\frac{d}{d X}\left(G \frac{d \bar{p}}{d X}\right)=\frac{d E}{d X}
$$

where

$$
\begin{aligned}
& G=\bar{h}^{2}\left(12+4 \bar{h} \bar{s}_{1}+4 \bar{h} \bar{s}_{2}+\bar{h}^{2} \bar{s}_{1} \bar{s}_{2}\right)+12 \bar{s}\left(\bar{k} \overline{l_{1}}+\bar{k} \bar{l}_{2}\right), \\
& E=-6 S \bar{s} X+6 \overline{s_{1}} \bar{h}\left(2+\bar{h} \bar{s}_{2}\right) .
\end{aligned}
$$

Equation (16) is known as dimensionless Reynolds's equation.

Since the pressure is negligible on the boundaries of the slider bearing compared to inside pressure, solving equation (16) under boundary conditions

$$
\bar{p}=0 \text { when } X=0,1 \text {. }
$$

The dimensionless film pressure $\bar{p}$ is obtained as:

$$
\bar{p}=\int_{0}^{X} \frac{E-Q}{G} d X,
$$

where

$$
Q=\frac{\int_{0}^{1} \frac{E}{G} d X}{\int_{0}^{1} \frac{1}{G} d X} .
$$

The load carrying capacity $W$ in dimensionless forms as

$$
\bar{W}=\frac{h_{1}^{2} W}{\eta A^{2} B U}=-\int_{0}^{1} X\left(\frac{E-Q}{G}\right) d X,
$$

where

$$
W=\int_{0}^{B} \int_{0}^{A} p d x d y
$$

\section{Results and Discussion}

Both porous plates inclined slider bearing with slip velocity and squeeze velocity lubricated by conventional fluid are examined to explore the possible effects on the bearing characteristic like load capacity.

The dimensionless load capacity $\bar{W}$ for various values of $\bar{l}_{1}$ and $\bar{l}_{2}$ are displayed in the Table 1 and 2 for various values of the following parameters :

$$
\begin{gathered}
\eta_{x}=0.64, m_{x}=0.81, \quad A=0.15(\mathrm{~m}), \\
h_{1}=0.05(\mathrm{~m}), \eta=0.012\left(\mathrm{kgm}^{-1} \mathrm{~s}^{-1}\right), \quad U=1.0\left(\mathrm{~ms}^{-1}\right),
\end{gathered}
$$




$$
\dot{h}=-0.005\left(\mathrm{~ms}^{-1}\right), a=2.0(\mathrm{~m}), k=0.0001 \mathrm{~m}^{2}
$$

Table 1 shows that the dimensionless load capacity increases when porous layer at both the ends are small. Other way we can say that the dimensionless load capacity increases when the porous layer at both the plates decreases.

It can be seen form Table 1 that for $\eta_{x}=m_{x}=0.81$ the dimensionless load capacity for $\bar{l}_{1}=\bar{l}_{2}=1.0$ is 0.199 , and for $\eta_{x}=m_{x}=0.64$ the dimensionless load capacity for $\bar{l}_{1}=\bar{l}_{2}=1.0$ is 0.202 . Thus the increase in dimensionless load capacity is approximately $1.5 \%$. For different and interchanging values of $\eta_{x}$ and $m_{x}$ at both the ends, there is no major difference in dimensionless load capacity as can be seen from column 3 and 4 of Table 1.

Table 1. Dimensionless load capacity for various combinations of $\bar{l}_{1}$ and $\bar{l}_{2}$ with respect to $\eta_{x}$ and $m_{x}$

\begin{tabular}{|c|c|c|c|c|c|}
\hline $\bar{l}_{1}$ & $\bar{l}_{2}$ & $\begin{array}{c}\eta_{x}=0.64 \\
m_{x}=0.81\end{array}$ & $\begin{array}{c}\eta_{x}=0.81 \\
m_{x}=0.64\end{array}$ & $\begin{array}{c}\eta_{x}=0.64 \\
m_{x}=0.64\end{array}$ & $\begin{array}{c}\eta_{x}=0.81 \\
m_{x}=0.81\end{array}$ \\
\hline 1.0 & 1.0 & 0.201 & 0.200 & 0.202 & 0.199 \\
\hline 5.0 & 5.0 & 0.109 & 0.109 & 0.110 & 0.109 \\
\hline 10.0 & 10.0 & 0.071 & 0.071 & 0.071 & 0.071 \\
\hline 20.0 & 20.0 & 0.042 & 0.042 & 0.042 & 0.042 \\
\hline 40.0 & 40.0 & 0.023 & 0.023 & 0.023 & 0.023 \\
\hline
\end{tabular}

Table 2. Dimensionless load capacity for interchanging sizes of $\bar{l}_{1}$ and $\bar{l}_{2}$ with respect to $\eta_{x}$ and $m_{x}$

\begin{tabular}{|c|c|c|c|c|c|}
\hline$\overline{l_{1}}$ & $\overline{l_{2}}$ & $\begin{array}{c}\eta_{x}=0.64 \\
m_{x}=0.81\end{array}$ & $\begin{array}{c}\eta_{x}=0.81 \\
m_{x}=0.64\end{array}$ & $\begin{array}{c}\eta_{x}=0.64 \\
m_{x}=0.64\end{array}$ & $\begin{array}{c}\eta_{x}=0.81 \\
m_{x}=0.81\end{array}$ \\
\hline 1.0 & 5.0 & 0.141 & 0.140 & 0.141 & 0.140 \\
\hline 5.0 & 1.0 & 0.141 & 0.140 & 0.141 & 0.140 \\
\hline
\end{tabular}

From Table 2, it is observed that, the dimensionless load capacity remains same if we interchange the size of the porous layers at both the ends.

\section{Conclusions}

Based upon the above formulation and results \& discussion, the conclusions can be drawn as follows for designing porous inclined slider bearing:

In order to have better load capacity it is suggested that

(1) The porous layer attached at both the ends should be thin and also the thickness remains same at both the ends.

(2) The values of porosity at both the ends should be small and equal.

(3) It should also be noted that interchange of the size of porous layers at both the ends will not effect on the load capacity.

\section{REFERENCES}

[1] H. Wu, Squeeze - film behavior for porous annular disks, Journal of Lubrication Technology, Trans. ASME, Series F, 92(4) (1970) 593-596.

[2] E. M. Sparrow, G. S. Beavers, I. T. Hwang, Effect of velocity slip on porous walled squeeze films, Journal of Lubrication Technology 94 (1972) 260-265.

[3] J. Prakash, S. K. Vij, Hydrodynamic lubrication of a porous slider, Journal of Mechanical Engineering Science 15 (1973) 232-234.

[4] R. C. Shah, N. I. Patel, Mathematical modeling of slider bearing of various shapes with combined effects of porosity at both the ends, anisotropic permeability, slip velocity, and squeeze velocity, American Journal of Computational and Applied Mathematics 2 (2012) 94-100.

[5] S.V.Kulkarni, V.Kumar, Lubrication equation for non-isotropic porous bearings considering slip velocity, IE(I) Journal - ME 56(1975)110-112.

[6] K. C. Patel, J. L. Gupta, Hydrodynamic lubrication of a porous slider bearing with slip velocity, Wear 85 (1983) 309-317.

[7] R. S. Gupta, P. Kavita, Analysis of rotation in the lubrication of a porous slider bearing: small rotation, Wear 111 (1986) 245-258.

[8] N.B. Naduvinamani , S. T. Fathima, S. Jamal, Effect of roughness on hydromagnetic squeeze films between porous rectangular plates, Tribology International 43 (2010) 2145-2151.

[9] F. Guo, P. L. Wong, M. Geng, M. Kaneta, Occurrence of Wall Slip in Elastohydrodynamic Lubrication Contacts, Tribol. Lett. 34 (2009)103-111.

[10] H. Urreta, Z. Leicht, A. Sanchez, A. Agirre, P. Kuzhir, G. Magnac, Hydrodynamic bearing lubricated with magnetic fluids, Journal of Physics: Conference Series 149 (2009) 012113

[11] R. C. Shah, M. V. Bhat, Ferrofluid lubrication equation for porous bearing considering anisotropic permeability and slip velocity, Indian Journal of Engineering \& Material Sciences 10 (2003) 277-281. 